\title{
Determination of Emitted X-Ray Tube Spectra by Means of a Calibrated Instrumental Setup
}

\author{
V. Rackwitz,* A. Warrikhoff,** U. Panne, ${ }^{* * * * *}$ and V.-D. Hodoroaba*
}

* BAM Federal Institute for Materials Research and Testing, 12200 Berlin, Germany

** rtw RÖNTGEN-TECHNIK DR. WARRIKHOFF GmbH \& Co. KG, 15366 Neuenhagen bei Berlin, Germany

*** Humboldt Universität zu Berlin, Department of Chemistry, 12489 Berlin, Germany

The accurate calculation of element concentration by means of standardless X-ray fluorescence (XRF) analysis requires besides accurate atomic and instrumental parameters the accurate knowledge of the excitation X-ray spectrum [1]. This paper deals with the determination of X-ray spectra emitted by side-window tubes in photons $\mathrm{eV}^{-1} \mathrm{msr}^{-1} \mathrm{nA}^{-1} \mathrm{~s}^{-1}$ by means of a calibrated instrumental setup.

Various algorithms for the calculation of the X-ray tube spectrum exist in the literature. For XRF the algorithms of (i) Pella et al. [2], (ii) Ebel [3] and (iii) Finkelshtein \& Pavlova [4] based on the socalled fundamental parameters and semi-empirical approaches are conventionally employed. A recent model based on accurate knowledge of a SEM/EDS (scanning electron microscope / energy dispersive X-ray spectrometer) instrumentation, especially of the spectrometer efficiency has been also referred to [5]. As an example X-ray spectra emitted by a Mo tube have been calculated with the four models and are shown together in Fig. 1. The calibrated SEM/EDS system enables to experimentally simulate the side-window X-ray tube. This is possible by tilting the sample in SEM as the target in the X-ray tube and by placing the EDS onto the horizontal port of the SEM. The corresponding Mo X-ray spectrum is included also into Fig. 1.

The results show that the various algorithms differ significantly in the spectral distribution and in the intensities as well. Therefore, in order to determine the real emitted X-ray tube spectrum a special setup consisting on an extremely long distance of $16 \mathrm{~m}$ between the X-ray tube and the X-ray spectrometer was built (Fig. 2). Otherwise, a conventional X-ray spectrometer would attain the saturation due to the very high photon fluxes (up to $10^{9}$ photons s $\mathrm{s}^{-1}$ ) emitted by such side-window Xray tubes.

The efficiency of the X-ray spectrometer was calibrated up to $50 \mathrm{keV}$. For this purpose an X-ray spectrum of a reference material (RM) measured by the spectrometer belonging to the setup was related to the emitted spectrum of the same RM measured with a calibrated X-ray spectrometer [6]. In order to enable the certification of X-ray tube spectra as an automated tool in the quality assurance for the X-ray tube manufacturers, a software package was developed, see. Fig. 3.

\section{References}

[1] J. Sherman, Spectrochim. Acta 7 (1955) 283.

[2] P. A. Pella et al., X-Ray Spectrom. 14 (1985) 125.

[3] A. L. Finkelshtein and T. O. Pavlova, X-Ray Spectrom. 28 (1999) 27.

[4] H. Ebel, X-Ray Spectrom. 28 (1999) 255.

[5] M. Procop and V.-D. Hodoroaba, Microchim Acta 161 (2008) 413. 
[6] V. Rackwitz et al., J. Anal. At. Spectrom. 24 (2009) 1034.

[7] Many thanks are due to Dr. M. Procop for helpful discussions and Mr. R. Gaens for performing measurements. The work is part of the project "MNPQ-Transfer" 23/07. V. Rackwitz acknowledges the support of the "BAM-Doktorandenprogramm".

FIG. 1. $30 \mathrm{kV}$ Mo X-ray tube spectra calculated with the models [2] - [5] and the spectrum measured with a calibrated SEM/EDS system (spectra are normalized to Mo K $\alpha$ intensity).
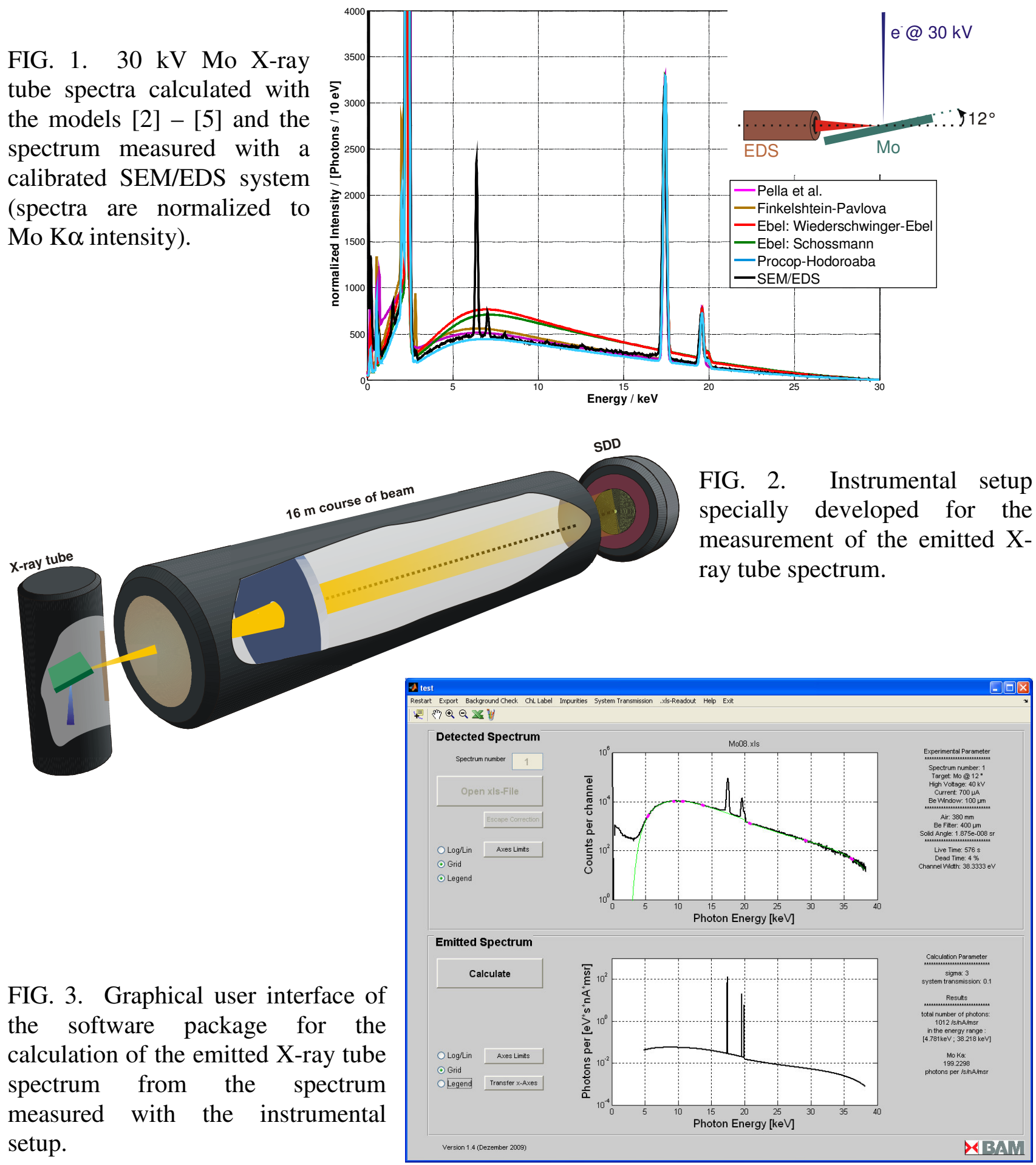